\section{PhRMA Establishes Dissolution Expert Team}

\author{
Peter Scott \\ Senior Scientist, QA Analytical Services, Method Services \\ AstraZeneca, 50 Otis St., Westborough, MA 01581
}

$\mathrm{n}$ the early days of dissolution, the need for control of variables led to studies which identified sources of error that are better defined and understood today. The field of dissolution is changing and growing as the science develops. Many dissolution techniques have improved, equipment standards are being harmonized, and analysts are educated and trained in the science of dissolution. The diversity of dosage forms is increasing and regulatory Guidelines are evolving. With the emergence of new technology there is an increased emphasis on better-controlled systems. All these factors indicate the need to study and evaluate the impact of such changes and developments on the testing community.

In light of the evolving technologies and new ideas in the dissolution community, the Pharmaceutical Research and Manufacturers of America (PhRMA) has created a Dissolution Experts Team with representatives from companies in the industry. The team is charged with keeping abreast of regulatory and compendial proposals that have an impact dissolution testing, and with coordinating efforts to influence developments.

As one of its current projects, the team has set a goal to organize studies and protocols to ensure better understanding of dissolution variables, their interactions, and their impact on the results of dissolution testing. The team is also evaluating the current state of dissolution calibration and investigating improvements to the system. The team is chaired by Mary Oates and includes the following members: Lee Bennett, Nina Cauchon, Patrick Crowley, Jean Gallery, Mary Ann Gorko, Al Kentrup, Greg Martin, Ngozi Okafo, David Rogers, Peter Scott, and Judy Shimek.

\section{Looking for qualified job applicants?}

Dissolution Technologies may be best place to advertise for qualified applicants for positions in the biopharmaceutics and dissolution area. If your company is interested, please contact the Managing Director at Vgray@dissolutiontech.com.

\section{The Dissolution} Solutions Network

In the developing field of pharmaceutical testing, there is a great reliance on dissolution testing, with limited resources available.

However, there is a new resource that has gotten the attention of many, The Dissolution Solutions Network, http://www.dissolutionsolutions.net/. It is the latest web site devoted to practical advice to dissolution analysts. The dissolution solutions network is a network of dissolution professionals dedicated to sharing collected knowledge in the advancing field of dissolution analysis. The site contains resources such as troubleshooting tips, calibration help, links, and a forum where both members and guest may discuss topics in organized "forum rooms" arranged in topics from Automation and Fiber Optics to Regulations and Biopharmaceutics.

Among other features is a web log, updated every Wednesday, including the latest news affecting the dissolution scientists. Additionally, the web site features recent articles by Vivian Gray on fiber optic dissolution testing, offering advice on evaluating the systems that are available and preparing to purchase a fiber optics system. It also features flexible XMLbased technology such as pages that adjust to a reader's screen size and pages that configure themselves to fit any printed page. The site is independently run by users and is also supported by advertising. 